\title{
Die Bedeutung der Masern in Sammelniederlassungen nach den im k. k. Flüchtlingslager Gmünd gemachten Wahrnehmungen.
}

\author{
Von \\ Dr. Josef Reder, \\ k. $k$. Oberbezirksarzt in Gimünd. \\ Mit 2 Abbildungen. \\ (Eingegangen am 10. August 1918.)
}

Daß in Kriegszeiten Seuchen aller Art aufzutreten pflegen, ist eine alte Erfahrung. Die durch die Kriegsverhältnisse bedingten Verschiebungen von großen Menschenmassen und ihre Unterbringung auf engem Raume in Unterkünften, die gewöhnlich die primitivste Reinlichkeitspflege unmöglich machen, prïdisponieren geradezu zum Auftreten infektiöser Krankheiten. Tatsächlich sahen wir denn auch gleich zu Beginn des großen Völkerringens die Infektionskrankheiten nicht nur unter den Truppen, sondern auch im Hinterlande, in Gefangenenund Flüchtlingslagern üppig in die Halme schießen. Begreiflicherweise wandte sich jedoch das Interesse des Publikums, der Sunitïtsbehörden und der Ärzte nur den, wenn ich so sagen darf, großen unter den Infektionskrankheiten, von denen man eine wesentliche Gefährdung der Volksgesundheit fürchtete, zu, deren man übrigens - und es darf dies als ein bleibendes Ruhmesblatt für die prophylaktische Medizin gebucht werden - fast allenthalben in verhültnismäßig kurzer Zeit Herr zu werden rerstand. In diesem Zusammenhange von den Masern zu sprechen, mag auf den ersten Blick ein parva componere magnis und müßig erscheinen, allein dic mit den Masern gemachten Erfahrungen, wenigstens soiveit sie meinen Wirkungskreis - das Flüchtlingslager Gmünd in Nieder-Österreich - betreffen, waren derart überraschend, daß sie wohl geeignet sein dürften, zu einer gründlichen Revision der althergebrachten Ansicht von der Harmlosigkeit der Masern zu führen.

Zur Orientierung sei gesagt, daß das Flüchtlingslager eine Sammelniederlassung war, die im Durchschnitt 20-25000 Flüchtlinge vorwiegend ukrainischer Nationalität beherbergte. Die Wohnungseinheit war die Baracke, die im wesentlichen in zwei Typen für den Flüchtlingsbelag in Betracht kam. Dic cine Type 
war ohne alle Unterabteilungen und bestand demnach aus einem einzigen mit Pritschenlagern versehenen großen Raume, in welchen der Längsachse der Baracke entsprechend eine ebenfalls dem Belage dienende Etagenpritsche eingebaut war. Eine solche Baracke beherbergte nicht selten 250-300 Personen. Es braucht nicht besonders betont zu werden, daß diese Type vom ärztlichen und speziell vom Standpunkte der Seuchenbekämpfungsmöglichkeit nicht diskutabel war (sie wurde übrigens nach den mit ihr gemachten ungünstigen Erfahrungen hinterher in 3 Abtcile für je 60 Personen unterteilt, wobei die Etagenpritschen weggelassen wurden). Bei der zweiten Type war die Baracke in vier Abteile unterteilt und jeder diescr Räume zur Unterbringung von 40-50 Personen bestimmt. Da es sich zunächst in erster Linie darum gehandelt hatte, die in großen Mengen anflutenden Flüchtlinge möglichst rasch unter Dach zu bringen, und weil man von Haus aus nur mit einer kurzfristigen Unterbringung rechnete, konnte den Forderungen der Hygiene bei diesen Barackenbauten zunächst nur in beschränktem Maße Rcchnung getragen werden. Wenn auch die zweiterwähnte Type wesentlich besser als die erste war, so waren doch die Baracken samt und sonders schließlich nichts anderes als Massenquartiere. Zu diesen Mängeln in den Wohnungsverhältnissen kam, daß das Flüchtlingslager hinsichtlich seines Belages keine stabile Masse war, daß es vielmehr infolgc beständiger An- und Abtransporte, die unvermeidlich waren - sie entzogen sich zumindest der ärztlichen Ingerenz -, nicht was es vi nominis sein sollte, ein Ort der Ruhe oder des Rastens, sondern, wenn auch nicht gerade eine Durehzugsstation, so doch sicher ein Ort lebhafter Unruhe und stark fluktuierender Bevölkerung war. Der Umfang dieser in den Belagsverhältnissen herrschenden Unruhe erhellt sehon daraus, daß während des dreijährigen Bestandes des Lagers mehr als 170000 Flüchtlinge durch das: selbe passierten. Daß dieses Moment die Seuchenbekämpfung besonders erschwerte, bedarf wohl keiner ausdrücklichen Frwähnung.

Die Masern wurden gleich mit den ersten Flüchtlingstransporten im Anfange des Jahres 1915 in das Lager eingeschleppt und erhielten sich mit einer solchen Hartnäckigkeit, dal bis Mitte März 1917 kein Tag verging, an dem es nicht Masernerkrankungen gegeben hätte. In den beiden ersten Monaten des Jahres 1915 spielten die Masern, von denen es damals im ganzen 50 Fällc, denen 5 Sterbefälle entsprachen, gab, keinerlei Rolle. Im März 1915 jedoch kam es. bereits zur ersten Epidemie, die vier Monate dauerte, und in welcher 628 Personen erkrankten; von diesen starben 286, so daß die Sterblichkeit dieser Epidemie $\mathbf{4 5 , 5 4} \%$ beträgt. Von der Epidemie waren ausschließlich Ukrainer befallen worden, da um diese Zeit keine andere Nationalität im Lager vertreten war. In den Monaten Juli und August 1915 traten die Masern lediglich in sporadischer Form (mit 38, bzw. 29 Erkrankungs- und 19, bzw. 13 Todesfällen) auf. Mit Mitte September änderte sich jedoch das Bild. Um diese Zeit erfolgten nämlich nach der Kriegserklärung Italiens große Antransporte von Südländern (Slowenen, Kroaten), die zahlreiche 
nach den im k. k. Flüchtlingslager Gmünd gemachten Wahrnehmungen. 357

Kinder mitbrachten, Transporte, in denen häufig gleich bei ihrem Einlangen masernkranke Kinder ausgehoben werden konnten. Mit Ende September 1915 (s. Abb.1) begann denn auch sofort die $\mathrm{z}$ wei te große Epidemi e. Die Kurve steigt steil an und erreicht in der ersten Woche Oktober - der Wochenzuwachs betrug 224 Fälle - ihren vorläufigen Höhepunkt, bält sich durch drei Wochen ungefähr auf dem gleichen Niveau, sinkt in der vierten Woche des Oktobers zwar erheblich $a b$, steigt aber sofort wieder rapid an, um mit Mitte November - Wochenzuwachs 646 Fälle - ihren höchsten Gipfel zu erreichen, von dem sie in der zweiten Novemberhälfte ebenso steil fast bis zum Nullpunkte

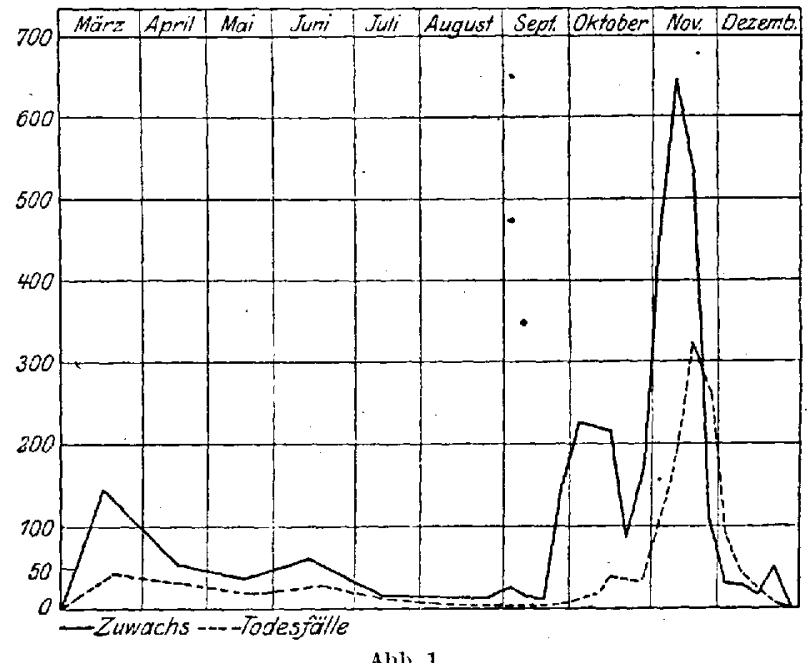

Abb. 1.

abfällt; in Dezember waren lediglich noch clie Ausläufer der Epidemie wahrzunehmen. Während dieser zweiten Epidemie erkrankten im ganzen 2411 Personen, von denen $\mathbf{1 1 6 3}$ der Seuche zum Opfer fielen. Die Mortalität dieser Epidemie betrug daher 48,23\%. Diese zweite Epidemie wütete ausschließlich unter den Südlündern. Die Gesamtzahl der im Jahre 1915 vorgekom menen Masernfälle (Epidemien und sporadische Fälle) belief sich auf 3198 Erkrankungen und 1469 Todesfälle:

Die Masernsterblichkeit dieses Jahres beziffert sich daher anf $45,93 \%$.

Noch mehr als das Jahr 1915 stand das Jahr 1916 (s. Abb. 2) im Zeichen der Masern. Während dieses Jahres herrschten die Masern eigentlich ununterbrochen in epidemischer Form, immerhin profilieren sich 
im Kurvenbilde im wesentlichen zwei Gruppen von Elevationen als Ausdruck vọn größeren Epidemien deutlich ab. Vom Jänner bis April 1916 verlief die dritte Masernepidemie mit 965 Erkrankungsfällen, die zu 369 Todesfällen Anlaß gab und demnach eine Sterblichkeit von $38,23 \%$ aufweist.

Von dieser dritten Epidemie waren wieder nur Südländer betroffen. Nach den gemachten traurigen Lrfahrungen wurden im Monate Mai die Südländer in andere für sie klimatisch günstigere Lager abgegeben. Dafür erfolgte ungefähr rom Juni an wieder ein lebhafter Antransport von ukrainischen Flüchtlingen. Sofort beginnt denn auch Mitte Juni

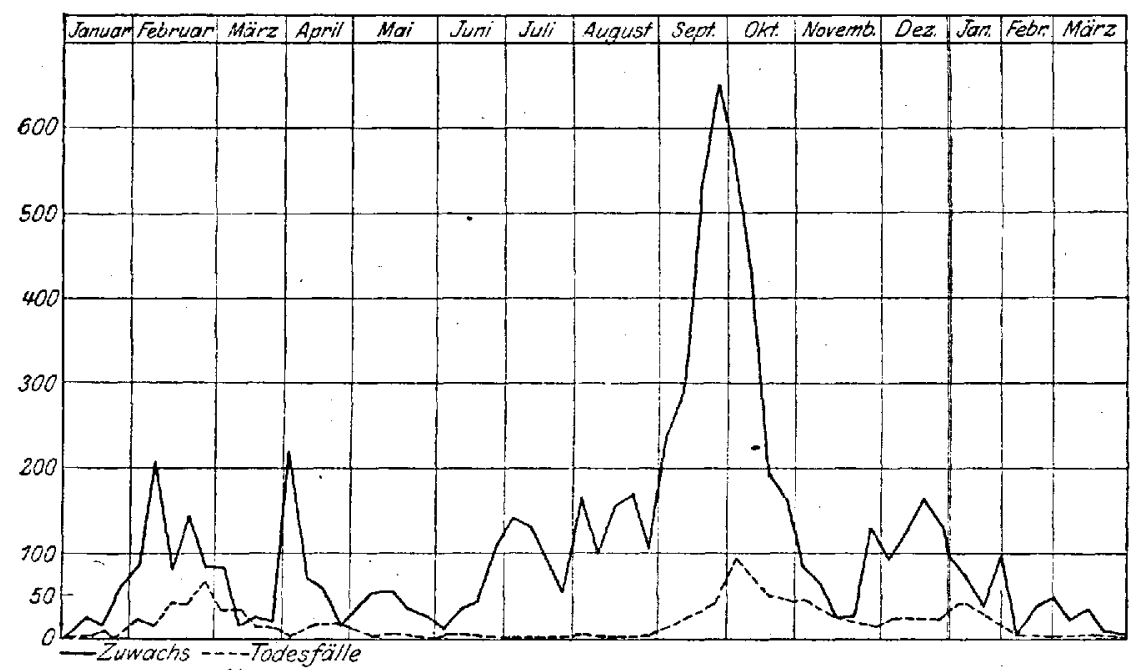

Abb. 2.

eine neue, die vierte Masernepidemic, welche die größte war. die je über das Lager gekommen ist. Die Epidemie wütete von Mitte Juni 1916 bis Mitte März 1917 und führte zur Erkrankung von 6011 Personen, vơn denen 934 an der Seuche zugrunde gingen. Die Mortalität dieser Epidemie beläuft sich daher auf $\mathbf{1 5 , 5 3} \%$. Diese vierte Epidemie hatte ausschließlich Ukrainer befallen, die Südländer waren, wie erwähnt, bereits aus dem Lager abtransportiert worden. Im ga nze n hat das Jahr 1916 zu 6543 Masernerkrankungen, denen 1140 Todesfälle entsprechen, geführt, so daß die Masernsterblichkeit dieses Jahres $17,42 \%$ ausmacht. (Die Masernfälle des Jahres 1917 beschränken sich ausschließlich auf die ersten drei Monate dieses Jahres und gehören organisch zu der letzten Epidemie des Vorjahres; es waren 
im ganzen 433 Erkrankungsfïlle, von denen 163 tödlich endeten.)Von Mitte März 1917 an war das Lager vollkommen masernfrei; seit dieser Zeit war aber auch eine Stabilisierung der Berölkerung insofern eingetreten, als von da ab keinerlei nennenswerte Antransporte von Flüchtlingen mehr erfolgt waren. Erst Mitte März 1918 sahen wir wieder einige Masernfälle jm Lager. Um diese Zeit wurden uns nämlich 6000 Rekruten zur Entlausung überwiesen, von denen 4000 gleich nach erfolgter Entlausung zu ihren Kadern zurückgeschickt wurden, während 2000 zu Quarantänezwecken vier Wochen im Lager zu verbleiben hatten. Sofort erkrankten von diesen Soldaten 25 an schweren Masern, denen 9 davon erlagen.

Tabelle 1.

\begin{tabular}{|c|c|c|c|c|c|c|c|c|c|}
\hline \multirow{3}{*}{ Kranklieit } & \multicolumn{8}{|c|}{ Zahl der Frkrankungen $\mathrm{E}$ und Todesfalle $\mathrm{T}$ : } & \multirow{3}{*}{ 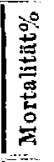 } \\
\hline & \multicolumn{2}{|c|}{1915} & \multicolumn{2}{|c|}{1916} & \multicolumn{2}{|c|}{1917} & \multicolumn{2}{|c|}{ Summe: } & \\
\hline & $\mathrm{E}$ & $\mathbf{T}$ & E & $\mathbf{T}$ & $\mathbf{E}$ & $\mathbf{T}$ & $\mathrm{E}$ & $\mathrm{T}$ & \\
\hline holera... . . . & - & - & 4 & 2 & - & - & 4 & 2 & 50 \\
\hline ern....... & 182 & 64 & 32 & 16 & 2 & 1 & 216 & 81 & \\
\hline fieber . . . & 902 & 142 & 154 & 31 & 48 & 9 & 1104 & 182 & 1 \\
\hline Trachom. . . & 38 & - & 41 & $-i$ & 882 & - & 967 & - & $\theta$ \\
\hline erie & 3 & - & 44 & 19 & 157 & 71 & 204 & 90 & 4 \\
\hline abdominalis . & 48 & 19 & 281 & 37 & 93 & 7 & 422 & 63 & \\
\hline rie. . . . . & 74 & 30 & 558 & 200 & 180 & 43 & 812 & 273 & \\
\hline $\operatorname{ach} .$. & 272 & 140 & 1853 & 352 & 118 & 20 & 2243 & 512 & \\
\hline usten. . & 52 & 42 & 129 & 30 & 17 & 8 & 198 & 80 & 40 \\
\hline & 45 & - & 112 & - & 64 & - & 221 & - & $\theta$ \\
\hline Rotlauf ....... & 117 & 15 & 286 & 21 & 137 & 15 & 540 & 51 & \\
\hline Sumue olme Masern. & 1733 & 452 & 3494 & 708 & 1698 & 194 & 6925 & 1334 & 19 \\
\hline Masern & 3198 & 1469 & 6.543 & $11+0$ & $43 ; 3$ & 163 & $|10174|$ & 2872 & 21 \\
\hline
\end{tabular}

Betrachtet man die Masern nach der Zahl der Erkrankungsfälle. so zeigt ein Blick auf die Tab. 1, daß die einzelnen Infektionskrankbeiten in dieser Beziehung nicht nur nicht annähernd an die Masern heranreichen, daß viclmehr wenigstens in den Jahren 1915 und 1916 die $Z$ ahl der Masernfälle nahezi dop pelt so groß ist, als die Erkrankungen an allen anderen Infektionskrankheiteu zusammen genommen. Das Gesamtergebnis der Masernerkrankungen aus den Jahren 1915--17 bessert sich (infolge der geringen Beteiligung des Jahres 1917 an der Masernmorbidität) allerdings insofern, als die Gesamtzahl der Masernerkrankungen - 10174 - die Summe der Erkrankungsfälle aller Infektionskrankheiten der drei Jahre zusammengenommen - 6925 - nur mehr um etwas mehr als 3000 Fülle übertrifft. 
Die zweite Epidemie des Jahres 1915 zeigte die ganz respektable Erkrankungsziffer 2411, die vierte Epidemie erreichte sogar die gewaltige Zahl von 6011 Fällen und blieb demnach hinsichtlich der Zahl nicht hinter der berühmten von Panum beschriebenen Epidemie auf den Faröern zurück.

Die Masern sind bekanntlich bereits im Initialstadium, das auch das katarrhalische, das Prodromal- oder das Stadium der Enantheme heißt, also bereits 3-4 Tage vor Ausbruch des Exanthems im hohen Maße ansteckend; nicht selten sind die Symptome dieses Stadiums so wenig deutlich, daß es gar nicht erst des Barackenmilieus bedarf, um sich die Schwierigkeiten der Diagnose vor Augen zu stellen. Die

Tabelle 2.

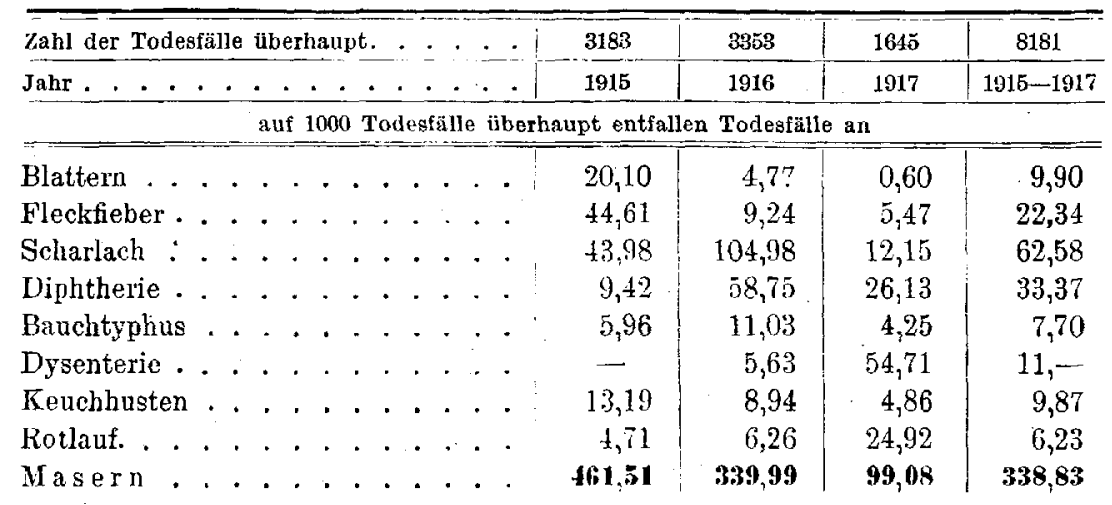

ärztliche Visite in einer überfüllten Baracke, in welcher es einem Ameisenhaufen gleich wimmelt, wird bei der mangelhaften Beleuchtung und bei dem geringen Entgegenkommen der. Insassen, die wenigstens in der Anfangszeit allen ärztlichen Bestrebungen nur mit dem größten Mißtraken gegenüberstanden, und die es häufig genug verstanden, sich mit allen erdenklichen Mitteln der Untersuchung zu entziehen, gerade dort, wo es sich um diagnostische Feinheiten, wie die Erkennung der Koplik schen Flecke, handelt, oft genug erfolglos sein. Was aber das Übersehen eines einzigen derartigen Falles in einer Baracke bedeutet, braucht nicht erst lebhaft geschildert werden, ein einziger solcher. Fall reicht hin, um alle Kinder dieser Baracke, sofern sic noch nicht gemasert haben, zu infizieren. Die gigantischen Maseruerkrankungsziffern des Flüchtlingslagers haben daher weiter nichts Erstaunliches an sich, sie erklären sich zwanglos aus den Besiedelungsverhältnissen, welche der Masseninfektion geradezu die Wege ebneten. 
nach den im k. k. Flüchtlingslager Gmünd gemachten Wahmehmungen. 361

Die Todesfälle an Masern beliefen sich im Jahre 1915 a uf 1469; ihre Zahl war dreimal so groß wie die Summe der Todesfälle aller anderen Infektionskrankheiten, i m J a hre 1916 war die Sterbeziffer der Masern $1140 \mathrm{um}$.mehr als 300, und die Gesamtzahl der Masern. todesfälle in den Jahren $1915-1917$ mit $2 \gamma \% 2$ mehrals doppelt so groß, wie sämtiche von allen Infelstionskrankheiten zu: sammen verursachten Todesfälle. Die Bedeutung der Masern für die allgemeine Mortalität springt noch mehr in die Augen, wenn man sie, in ihrem Verhältnis auf 1000 Todesfälle überhaupt berechnet, mit den anderen Infektionskrankheiten vergleicht (s. Tabelle 2). Während beispielsweise, um nur die wichtigeren Infektionskrankheiten herauszugreifen, im Jahre 1915 auf 1000 Todesfälle überha pt rund 20 Todesfälle an Blattern; 44 an Fleckfieber, 43 an Scharlach, 9 an Diphtherie entfallen, kommen von den Masern 461, i m Jahre 1916 bei 5 Todesfällen an Blattern, 9 an Fleckfieber, 104 an Scharlach und 58 an Diphtherie, 339 Sterbefälle an Masern auf 1000 Todesfälle überhaupt. Im Summarergebnisse (1915-1917) fallen 9 Todesfälle an Blattern, 22 an Fleckfieber, 62 an Scharlach, 33 an Diphtherie, dagegen 338 an Masern auf 1000 Todesfälle iberhaupt.

Sichtet man die Maserntodesfälle nach dem Alter (s. Tabelle 3), so ergibt sich, daß von den $27 \% 2$ Sterbefällen $1394=50,29 \%$ auf die beiden ersten Lebensjahre, das Säuglingsund Kleinkindesalter, $1132=\mathbf{4 0 , 8 3} \%$ auf das Spielalter (drittes bis inkl. sechstes Jahr) und nur $198=\mathbf{7}, 14 \%$ auf das Schulalter entfallen. Das erste und zweite Lebensjahr verhält sich hinsichtlich der Zahl der Sterbefälle fast vollkommen gleich; betrachtet man jedoch die

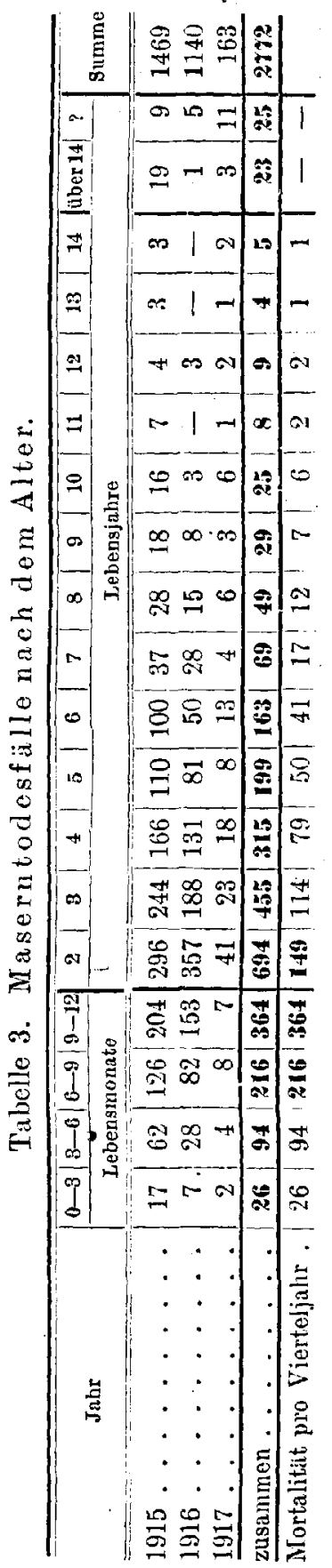


Sterblichkeitsverhältnisse des ersten Lebensjahres gesondert nach den einzelnen Vierteljahren, so zeigt sich, daß das erste Vierteljahr mit seinen 26 Sterbefällen hinsichtlich der Masernsterblichkeit nahezu keine Bedeutung hat, da $\beta$ aber auch das zweite Vierteljahr mit im ganzen 94 Erkrankungen an der allgemeinen Masernsterblichkeit nur in verhältnismäßig sehr beschränktem Maße teilnimmt. Wem nun auch infolge der überstürzten Tätigkeit, welche die Masernepidemien, mit denen im Jahre 191.5 eine große Fleckfieber- und im Jahre 1916 eine sehr erhebliche Schärlachepidemie einherging, mit sich brachten, die Zahl der Erkrankten der einzelnen Alterskategorien, wie es wünschenswert gewesen wäre, nicht ermittelt worden ist, so bestätigen doch die Mortalitätsziffern gerade des ersten und zweiten Lebensvierteljahres deutlich die Richtigkeit der Angaben Filatows, Jochmanns, Feers, Thomas', Jürgensens, Pfeilstickers u. a. daß die Empfünglichkeit für Masern bei Kindern bis zum sechsten Lebensmonat ungemein gering ist. Im dritten und vierten Lebensquartale mit 216, bzw. 364 Todesfällen erscheint dagegen die durchschnittliche Disposition des Kindesalters bereits erreicht. Vóm zweiten Lebensjahre an fallen die Todesfälle im Spiel- und hinein in das Schulalter gradatim staffelförmig derart ab, daß das Schulalter für die Mortalität nahezu leine Bedeutung mehr hat.

Tabelle 4. Maserntodesfälle nach Komplikationen.

Gesamtzahl der Maserntodestalle . . . . . . . . 2772

Davon an Komplikationen gestorben......... $2093=\mathbf{2 0 9 5}, \mathbf{5 0} \%$ ind zwar an:

Bronchopneumonie . . . . . . . . . . $17830=61,68 \%$

Lungentuberkulose. . . . . . . . . . . $189=6,89 \%$

Bronchiolitis .............. . $86=\mathbf{3 6}, \mathbf{1 0} \%$

Enteritis . . . . ............ $40=1,44 \%$

Nephritis . . . . . . ........ . $6=0,21 \%$

Meningitis tbc. .............. . . $11=0,39 \%$

Noma ................ . . 2

Mastoiditis ................ . 1

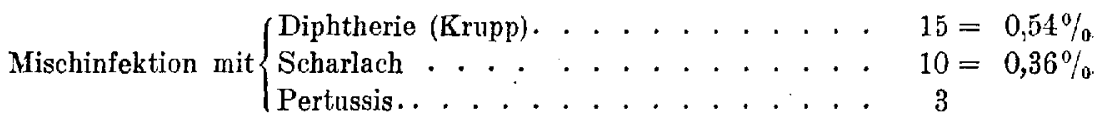

Die weitaus überwiegende Mehrzahl der Kinder ist nicht an der Infektion selbst, sondern an Komplikationen (s. Tab. 4) zugrunde gegangen. Von den $\mathbf{2 7 7 2}$ an Masern gestorbenen Kindern sind nämlich $2093=75,50 \%$ durch Komplikationen dahingerafft worden. Dic bei 
nacb den im k. k. Flüchtlingslager Gmünd gemachten Wahrnehmungen. 363

weitem dominierende Rolle fiel dabei den Komplikationen des Respirationstraktes zu, unter denen die Bronchopneumonie mit der erschreckenden Ziffer von $1730=61,68 \%$ an der Spitze steht. Es handelte sich dabei in der Regel um disseminierte und pseudolobäre Bronchopneumonien, die häufig an der Peripherie der Entzündungsherde eine eigentümliche nekrotische Einschmelzung des Gewebes von fast dissezierendem Charakter aufwiesen. Eigentliche kruppöse Pneumonien waren verhältnismäßig selten. Die Bronchopneumonie war ohne $Z$ weifel die alles beherrschende Komplikation, der gegenüber alle anderen in den Hintergrund traten. Immerhin ist auch die Tuberkulose mit 189 Fällen $=6,89 \%$ der Maserntodesfälle an der Sterblichkeit beteiligt; hierbei sind nur jene Tuberkulosefälle in Rechnung gezogen, welche in Form der Miliartuberkulose, der käsigen Pneumonie, der Pleuritis, der Miliar- und der Hilusdrüsentuberkulose der Masernerkrankung unmittelbar subsequierten. . Die Meningitis tbc. mit 11 Todesfällen $(=0,39 \%$ der Maserntodesfälle) ist dabei nicht eingerechnet. Die Disposition der Masern für Tubcrkulose ist hinlänglich bekannt und ist, wie es die von Preisich gefundene Tatsache des temporären Fehlens der kutanen Tuberkulinreaktion während des Stadiums der Florition wahrscheinlich macht, dadurch bedingt, daß der masernkranke Organismus, dessen Antikörper nicht zur Entfaltung gelangen können, der Infektion wehrlos gegenübersteht. Von weiteren Komplikationen des Respirationstraktes ist schließlich noch die Bronchiolitis mit 86 Fällen $=3,10 \%$ aller Maserntodesfälle zu erwähnen. Die Enteritis war mit 40 Todesfällen $=1,44 \%$ aller Masernsterbefälle vertreten. An Nephritis, einer bei Masern überhaupt nicht hänfigen Komplikation, sind im ganzen nur 6 Kinder zugrunde gegangen. Mischinfektionen mit Diphtherie (Krupp) kamen in 15, mit Scharlach in 10 und mit Pertussis in 3 Fällen zur Beobachtung. Endlich sind noch zwei Fälle von Noma anzuführen. Bei einem auffallend hohen Prozentsatze der verstorbenen Kinder wurde übrigens am Sektionstische Hyperplasie der lymphatischen Organe festgestellt, eine Anomalie, die erfahrungsgemäß die Resistenz gegen Infektionskrankheiten überhaupt herabzusetzen pflegt.

Die Mortalität der Masern ist im allgemeinen nach den äußeren Umständen, unter denen sie auftreten, und, nach Epidemien außerordentlich verschieden. Sie schwankt (s. Tab. 5, die eine Zusammenstellung der bekannteren Masernepidemien gibt) zwischen $1,7 \%$ in der Münchener Epidemie von 1860 (Ranke) und 30,0\% der Masernfälle der Berliner Charité der Jabre 1888-1890 (Henoch). Jedenfalls kann die Sterblichkeit in der Privatpraxis nicht mit jener in gewissen 
Epidemien und schon gar nicht mit der gelegentlich bei Spitalbehandlung beobachteten verglichen werden, die als ,Hospitalmortalität" seit jeher in üblem Rufe steht. So hat z. B. Fürbringer auf seiner Abteilung Friedrichshain in Jena in den Jahren 1886-1890 eine Sterblichkeit von $22,6 \%$ - i. J. 1886/87 sogar $30,4 \%$ - Mo nti im Kinderspitale in Wien von 1864-1867 eine Mortalität von $26,34 \%$ und Henoch in der Charité, wie bereits erwähnt, sogar 30,3\% Sterblichkeit gehabt. Laveran sieht als Ursache der hohen Spitalsterblichkeit dic verdorbene Spitalluft an, während Fürbringer wohl mit mehr Recht die Art

Tabelle 5.

Sterblichkeit in einigen bekannten Masernepidemien.

\begin{tabular}{|c|c|c|c|c|}
\hline ort & Zeit & $\begin{array}{l}\text { Zahl der } \\
\text { Krankheits- } \\
\text { fälle }\end{array}$ & $\begin{array}{c}\text { Mortalität } \\
\% \\
\%\end{array}$ & Autor \\
\hline ........ & $1827-28$ & 2100 & 1,8 & Faber \\
\hline Würzburg (Kinderklinik) . . . . & $1842-71$ & 851 & 4,5 & Voit \\
\hline Frankfurt . . . . . . & $1860-61$ & 1788 & 2,57 & SpieB \\
\hline Meerane .......... & 1861 & 1763 & 3,5 & GeiBler \\
\hline Kiel . . . . . . . . & 1860 & 573 & 6,8 & Bartels \\
\hline Greifswald . . . & 1861 & 311 & 6,8 & $\begin{array}{l}\text { ZiemBen- } \\
\text { Krabler }\end{array}$ \\
\hline Würzburg (Kinderklinik) . . . & 1863 & $?$ & 10,5 & $\begin{array}{l}\text { zit. nach } \\
\text { Thomas }\end{array}$ \\
\hline Niederösterreichische Epidemien . & $1862-63$ & $?$ & $2,29-6,29$ & Karajan \\
\hline Stuttgart . . . . . . . & $1852-65$ & 380 & 1,8 & Köstlin \\
\hline Wien (Kinderspital) . . . . . . & $1864-67$ & 372 & 26,34 & Monti \\
\hline München . . . . . . . & 1869 & 1907 & 1,7 & Ranke \\
\hline...... & ? & 129 & 9,1 & Brown \\
\hline Tübingen . . . . . . . . & $1874-93$ & 868 & 6,1 & Jurgensen \\
\hline Zolkiew. . . . . . . . . & 1880 & 1519 & 13,0 & Seidl \\
\hline Würzburg . . . . . . . . . & 1883 & 1896 & 8,1 & Breyer \\
\hline Jena (Friedrichshain) . . . . . & $1886-90$ & 453 & 22,6 & Fürbringer \\
\hline Berlin (Charité) . . . . . & $1888-90$ & 294 & 30,3 & Mennoch \\
\hline Heidelberg . . . . . . . . . & 1888 & $461^{\circ}$ & 6,7 & Emden \\
\hline
\end{tabular}

seines Materials - „fast alle Gestorbenen waren Jammerkinder, die bereits nit schweren Komplikationen eingeliefert wurden" - zur Erklärung heranzieht. Im Mittel beträgt die Sterblichkeit der Masern nach Jochmann und Henoch $6 \%$. Epidemien, wie jene in Leith mit 9,1\% (Brown) oder in Zolkiew mit 13\% (Seidl) Mortalität, rechnet Tho mas schon zu den schweren. Allen diesen Angaben gegenüber stellen sich die Zahlen unserer Epidemien (s. Tab. 6) leider als Rekordziffern dar. Eine Masernmortalität von 45,93\%, wie wir sie im Jahre 1915 hatten, bzw. eine Sterblichkeit von 45,54\%, wio sie die erste ukrainische Epidemie zeigte, und gar ron $48,23 \%$, wie sie der zweiten großen Epidemie des Jahres 1915 eigen war, ist epidemiologisch fast etwas Unerhörtes. Nur Thomas crwähnt eine Epidemie, die eine noch höhere Mortalität, nämlich 50\%, gehabt haben soll; 
nach dẹn im k. k. Flüchtlingslager Gmind gemachten Wahrnehmungen. 365

es war dies eine Epidemie, die im .Jahre 1856 in Lippe in Mittelungarn geherrscht hat, für welche jedoch dic Ziahl der Erkrankten bei Thomas nicht ausgewiesen erscheint. Im Jahre 1916 hatte die dritte Südländerepidemie noch cine Sterblichkeit von $38,23 \%$, die vierte größte Masernepidemie des Lagers überhaupt, an der nur Ukrainer beteiligt waren und die bis Mitte März 1917 reichte, dagegen ane Sterblichkeit von nur 15,53\%. In der ganzen dreijährigen Funktionsperiodè des Lagers ergab sich bei 10174 Frkrankungen mit 2772 Todesfällen eine Masernsterblichkeit von $\mathbf{2 7 , 2 4} \%$ im Durchschnitt. Die Masern wurden, was Bösartigkeit anbelangt, nur von der Dysenterie mit 44,11\% Todesfiullen, von den Blattern mit $37,5 \%$ und von der Diphtherie mit $33,22 \%$ übertroffen.

Tabelle 6.

Masernepidemien im k. k. Flüchtlingslager Gmünd.

\begin{tabular}{|c|c|c|c|c|c|c|}
\hline \multirow[t]{2}{*}{ Jahr } & \multirow[t]{2}{*}{ Nr. } & \multirow{2}{*}{$\begin{array}{c}\text { Jahreszeit } \\
\text { Monate } \\
\end{array}$} & \multicolumn{2}{|c|}{ 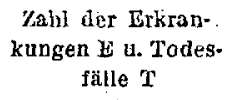 } & \multirow{2}{*}{$\begin{array}{c}\text { Mortalitiat } \\
\% \\
\end{array}$} & \multirow[t]{2}{*}{ Nationalität } \\
\hline & & & $\mathrm{E}$ & $T$ & & \\
\hline \multirow{4}{*}{1915} & 1 & III.-VI. & 628 & 286 & 45,54 & Ukrainer \\
\hline & 2 & IX:-XII. & 2411 & 1163 & 48,23 & Südlitnder \\
\hline & $\begin{array}{l}\text { sporadische } \\
\text { Fälle }\end{array}$ & $\begin{array}{l}\text { I.-Ir. } \\
\text { VII., VIII. }\end{array}$ & 159 & 20 & 12,57 & Ukrainer \\
\hline & & I.-XII. & 3198 & 1469 & 45,93 & \\
\hline \multirow{3}{*}{1916 . } & 3 & $1 .-\mathrm{IV}$ & 965 & 369 & 38,23 & Sudländer \\
\hline & 4 & VI.-XII. & 5578 & 771 & 13,82 & Ukrainer \\
\hline & & I. - XII. & 6543 & 1140 & 17,42 & \\
\hline \multirow{2}{*}{1917 . } & $\begin{array}{c}4 \\
\text { (Fortsetzung) }\end{array}$ & I.-III. & 433 & 163 & 37,64 & Ukrainer \\
\hline & $\begin{array}{c}4 \\
\text { (total) }\end{array}$ & $\begin{array}{l}\text { VI._XII. } 1916 \text {, } \\
\text { I.-III. } 1917\end{array}$ & 6011 & 934 & 15,53 & Ukrainer \\
\hline $1915-1$ & & & 10174 & 2772 & 27,24 & \\
\hline
\end{tabular}

Wir haben es natürlich gleich vom Anbeginn an nicht an Bemühungen fehlen lassen, die Masernepidemien nach Kräften zu bekämpfen. Panum hält es zwar in diesem Belang für ganz unzweifelhaft, daß Absperrung das sicherste Mittel sei, um die Ausbreitung der Masern zu verhindern. Dies mag nun vielleicht für die Verhältnisse auf den weltentlegenen Faröern zutreffen, in einem Flüchtlingslager darf man sich aber von derlei Absperrungen keinerlei Erfolg versprechen. Alle Absperrmaßnahmen der verseuchten. Baracken und auch der Versuch, durch Absonderung der Kontaktpersonen, soweit sie im Kindesalter standen, den Masern entgegenzutreten, haben vielmehr fehlgeschlagen. Dies erhellt am deutlichsten daraus, daß im Jahre 1916, trotzdem der ganze sanitäre Apparat mit viel größerer. Präzision als im Vorjahre funktionierte, die Zahl der Masern von 3198 im Jahre 1915 auf 6543, also auf mehr als das Doppelte, angestiegen war. Der Kampf gegen die Zahl hat sich also jedenfalls als vergeblich erwiesen. Trotzdem jedoch 
wurde die Flinte nicht ins Korn geworfen. Im Gegenteil! Erfahrungsgemäß gelingt es nicht selten, die Ansteckùng der Kontaktpersonen zu verhindern, wenn man ein masernkrankes Kind gleich im Beginn des Prodromalstadiums absondert, während Absonderung erst bei bereits beginnendem Ausschlag regelmäßig zu spät kommt (Feer). Es wurde daher die ganze sanitäre Arbeit in den Barackén, wo Masern aufgetreten waren oder erwartet wurden, nun darauf konzentriert, die Masern möglichst frühzeitig schon im Initialstadium festzustellen und sie bereits in diesem Stadium aus der Baracke weg- und in Spitalsbehandlung zu bringen. Die Kinder dieser Baracken wurden zweimal täglich gemessen und genau auf Prodromalsysmptome geprüft. Jedem Falle, der Temperaturerhöhung, Bronchitis, Coryza oder conjunctivalo Rötung zeigte, wurde die größte Aufmerksamkeit geschenkt. Namentlich aber wurde mit großem Eifer und methodisch nach Koplikschen Flecken gefahndet, worin Ärzte, Mediziner und Fürsorgeschwestern sich alsbald die erforderliche Routine aneigneten. Jedes Kind, das Kopliksche Flecken zeigte, wurde in das Maserńspital, jedes Kind, das bei Temperaturerhöhung Bronehitis, Schnupfen oder Bindehautkatarrh aufwies, in das Quarantänespital abgegeben. In den Baracken selbst, wo Masern gehäuft auftraten, wurde natürlich auch der allgemeinen Reinlichkeitspflege ein besonderes Augenmerk zugewendet (täglich feuchtes Aufwischen des Fußbodens, Lüftung der Baracken, der Strohsäcke und Bettsorten, 'einmal wöchentliches Ausspritzen der Baracke mit Kalkmilch oder mit Lysollösung).

Gestützt auf die Wahrnehmungen in der großen Epidemie des Jahres 1915 wurde weiter streng darauf geachtet, daß in den Spitälern Masernfälle mit Komplikationen, namentlich solche mit den gefürchteten Bronchopneumonien, von den nicht komplizierten Fällen vollkommen getrennt und möglichst in kleinen Räumen, wozu sich die Extrazimmer in den Infektionsspitälern und auch Abteile der sogenannten Waldbaracken besonders eigneten, untergebracht wurden.. Daß auch für richtige. Beheizung und Belüftung der Spitäler Vorsorge getroffen wurde, ist selbstverständlich. Das täglich feuchte. Aufwischen des Fußbodens speziell in jenen Räumen, wo komplizierte Fälle lagen, das Abwischen der Nachtkästchen mit Lysollösung und das wiederholte Ausspritzen dieser Räumlichkeiten mit Kalkmilch - eine Prozedur, die, während die Kranken vorübergehend in einem anderen Raume untergebracht wurden, bei Benutzung praktischer Handpumpen in kurzer Zeit durchführbar ist - war bei der bereits angedeuteten infektiösen Natur dieser Komplikationeu sicherlich nicht wertlos. 
nach den im k. k. Fluchtlingslager Gmund gemachten Wahrnehmungen. 367

Um die durch die überstandenen Masern geschwächten Kinder gegen Nachkrankheiten namentlich des Respirationstraktes nach Möglichkeit zu schützen, erschien es am Platze, sie nach dem Rate Ciepanowskis; der sich um die Bekämpfung der Masern in Lager besonders verdient machte, tunlichst lange von dem Barackenmilieu fern und in entsprechender Obsorge zu halten, um so mehr, als gerade zur Zeit der großen Epidemien die Witterungsverhältnisse, wie erwähnt, die denkbar ungünstigsten waren. Als Norm galt, komplizierte Fälle nach Maßgabe der Notwendigkeit, nicht komplizierte aber wenigstens noch 14 Tage nach der Entfieberung im Spital zu behalten. Weiter schonungsbedürftige Kinder wurden schließlich noch in eigenen Rekonvaleszentenheimen und bei besserer Verpflegung nach Erfordernis noch eine Zeitlang gehalten.

Vergleicht man die beiden großen Epidemien der Jahre 1915 und 1916 miteinander, so verliefen beide so ziemlich unter denselben ungünstigen Witterungsverhältnissen. Die Epidemie des Jahres. 1915 wütete fast ausschließlich unter Südländern, jene des Jahres 1916 dagegen betraf nur Ukrainer. Diese sind zwar nach unseren Erfahrungen zweifellos ihrer Anlage nach weit resistenter gegen Infektionen als die Südländer. Auch soll ohne weiteres zugegeben werden, daß in beiden Epidemien vielleicht die Virulenz der Erreger, der Genius epidemicus, verschieden gewesen sein kann, trotzdem ist der Abfall der Mortalität von 48,23 auf 15,53 ein so bedeutender und in die Augen fallender, daß er aus den angedeuteten Momenten allein nicht befriedigend erklärt werden kann. Wir sind vielmehr fest überzeugt, da 3 , wenn es auch nicht möglich war, die Ausbreitung der Epidemien zu beeinflussen, wir doch durch Entfaltung des geschilderter, freilich recht mühsamen Apparates und insbesondere durch Beobachtung einer indjviduellen Prophylaxe auf den Verlauf.des Einzelfalles und damit auch auf den Charakter der ganzen Epidemie selbst günstigen Einfluß zu nehmen imstande waren.

Zusammenfassend möchte ich zu folgendem Ergebnisse kommen:

1. Die Masern, im Familienverbande eine so harmlose Krankheit, daß3 sie von der allgemeinen Anzeigepflicht ausgenommen worden ist, können in Massenniederlassungen, wie es ein Flüchtlingslager ist, hinsichtlich ihrer Bedeutung für die allgemeine Morbidität und Mortalität die wichtigste Rolle spielen.

2. Sie waren zufolge der exorbitanten Malignität, mit der sie sich in manchen Epidemien präsentierten, die gefährlichste und am meisten gefürchtete aller Infektionskrankheiten; mit den Verwüstungen, welche die Masern anrichteten, verglichen, erscheinen 
die Schäden, welche die anderen Infektionskrankheiten, die sog. hochvirulenten nicht ausgenommen, verursachten, schon wegen der verhältnismäßig .geringen Höhe, die deren Erkrankungs- und Sterbezahlen gewöhnlich erreichen, von untergeordneter Bedeutung.

3. Die Verheerungen, welche durch die Masern veranlabt wurden, betrafen, wenn man von dem ersten Lebenshalbjahre, das eine deutlich verminderte Disposition für die Masern hat, absieht, vorzugsweise das erste und zweite Lebensjahr, und nahmen für die Jahre des Spielalters staffelförmig ab; das Schulalter hatte in diesem Belang nur mehr geringe Bedentung. Die Kinder der Südländer erwiesen sich dabei der Infektion gegenüber weitaus weniger widerstandsfähig als die Ukrainer.

4. Trotzdem die Masern zu allen Jahreszeiten aufgetreten waren, kam es zu größeren Epidemien doch immer nur im Herbst und Winter bei kaltem und nassem. Wetter, so daß dessen Einfluß auf das Entstehen der Epidemien (Hirsch) augenfällig. war. Die bei diesen Witterungsverhältnissen bestehende größere Anfälligkeit für katarrhalische Erkrankungen des Respirationstraktes, der wohl als Eintrittspforte des Erregers angesehen werden darf, macht diese Erscheinung von Haus aus erklärlich; daß derart ungünstiges Wetter der Entstehung von Lungenkomplikationen besonders förderlich ist, ist eine alte und kaum von irgendeiner Seite bestrittene Erfahrung.

5. Das Maserngift war im Flüchtlingslager zweifellos ubiquitär; solange Transporte einlangten, waren Personen, die noeh nicht gemasert hatten, der Infektion verfallen.

6. Der beste Schutz gegen das Auftreten der Masern in Lager wäre gewesen, Antransporte von Flüchtlingen zu vermeiden, eine Forderung, die sich jedoch aus anderen, der ärztlichen Einflúßsphäre sich entziẹhenden Gründen nicht verwirklichen lieB.

7. Die Bekämpfung der Masern, selbst unter den ungünstigen. Verhältnissen eines Flüchtlingslagers, bietet Aussicht auf Erfolg, sie darf sich jedoch nicht auf nutzlose Absperrungsmaßnahmen der verseuchten Baracken beschränken, sondern hat ihr Augenmerk darauf zu richten, die kranken Kinder möglichst frühzeitig im Initialstadium auszuheben ünd sie noch frei von Komplikationen in die Spitalbehandlung zu bringen.

8. Die Masernerscheinen nur unter Beobachtung gewisser Kautelen hospitalisierbar, deren wichtigste die Unterbringung der Kranken nach dem Rate v. Pirquets in nicht zu großen Verbänden und die Absonderung der komplizierten von den nicht komplizierten 
nach den im k. k. F]uchtlingslager Gmund genachten Wahmehmungen, 369

Fällen ist. Die sanitätspolizeilichen Maßnahnen, die in einen Flüchtlingslager gegen Masernepidemien zu ergreifen wären, lassen sich demnach in folgender Weise formulieren:

1. Quarantänierung der Antransporte, nicht so sohr als Absperrmaßnahme, sondern wegen der Möglichkeit beständiger ärztlicher Perlustrierung.

2. Täglich zweimaliges Thermometrieren aller Kinder: dess betreffenden Autransports.

3. Zweimal tägliche Barackenvisite mit der besonderen Aufgabe, jedes cinzelne Kind hauptrsächlich auf Kopliksche Flecke, auf Coryza, Bronchitis und Conjunctivitis zu untersuchen.

4. Abgabe aller Kinder mit Koplikflecken in das.Masernspital, Abgabe aller Kinder mit Schnupfen, Bindehautkatarrh, Bronchitis oder auch nur mit einem oder dem anderen dieser Symptome, besonders wenn gleichzeitig erhöhte Temperatur gefunden wird, in das Quarantänespital.

5. Reinlichkeitspflege in den Baracken fouchtes Aufwischen des Fußbodens, Lüftung der Baracken, der Strohsäcke und Bettdecken und einmal wöchentliches Ausspritzen der Baracke mit Kalkmilch).

6. Trennung der mit Komplikationen besonders mit Lungonentzündung behafteten Kinder im Spital von den unkomplizierten Fällen, Unterbringung der Kranken, unbedingt aber jener mit Komplikationen tunlichst nicht jn großen Krankensälen, sondern in kleineren Abteilen.

7. Richtige Temperierung und Lüftung der Krankenzimmer, täglich feuchtes Aufwischen des Fußbodens mit Sodalösung namentlich in jenen Räumen, wo die komplizierten Fälle liegen, für welche Räume sich auch wenigstens wöchentlich eimmaliges Ausspritzen mit Kalkmilch dringend empfiehlt. Bei dem häufigen infektiösen Charakter der Masernpneumonien kann diese Maßnahme, wiewohl eine Desinfektion bei Masern gesetzlich nicht vorgeschrieben ist, nicht als Ausfluß entbehrlicher Vielgeschäftigkeit angesehen werden.

8. Nicht zu frühzeitiges Entlassen der Genesenen aus dem Spital, uamentlich bei ungünstiger Witterung, um das Auftreten von späten Komplikationen seitens des Respirationstraktes zu vermeiden. Ohne Komplikationen verlaufene Fälle wären noch mindestens 14 Tage, von der Entfieberung an gerechnet, in Spitalpflege zu halten vund ärztlich zu überwachen. 